\title{
Patterns of oral nutritional support prescription in hospital in-patients: comparison of dietetic versus non-dietetic prescriptions
}

\author{
E. Walton ${ }^{2}$, M. O’Donnell ${ }^{1}$ and C. T. Soulsby ${ }^{1}$ \\ ${ }^{1}$ Barts and the London NHS Trust, Royal London Hospital, Whitechapel, London E1 1BB, UK and ${ }^{2}$ London Metropolitan \\ University, School of Human Sciences, 166-220 Holloway Road, London N7 8DB, UK
}

NICE guidance recommends that nutrition support in adults (oral, enteral or parenteral) should be: considered in patients who are malnourished or at risk of malnutrition, the prescription should be carried out by healthcare professionals who are skilled and trained in nutritional requirements and should take into consideration energy, protein, fluid, electrolyte, mineral, micronutrients and fibre needs ${ }^{(1)}$. At the Royal London Hospital, in-patient oral nutritional support (ONS) is prescribed by non-dietetic staff (nursing and medical staff) as well as by dietetic staff.

The aim of this audit was to compare patterns of ONS prescribing with NICE guidance. A prospective audit was carried out for 5 consecutive working days on 8 wards (medical, surgical and trauma). All patients were included unless they were nil by mouth (NBM). The dietetic auditor recorded rates of NST completion by ward staff, number of nutritional supplements taken in the previous $24 \mathrm{~h}$ and who had prescribed them and then completed a Nutrition Screening Tool (NST) on all patients. The NST was a modified Birmingham Heartlands tool ${ }^{(2)}$ assessing BMI or MUAC, appetite, ability to eat and a stress factor; completion of an NST by ward staff was taken as evidence that food intake had been considered by non-dietetic staff.

One hundred and thirteen patients were identified, 10 patients were excluded because they were NBM/receiving total enteral/parenteral nutrition and a further 8 patients could not be screened by the dietetic auditor as they were either off the ward or unwilling to participate leaving 95 patients. The NST found 9 patients (10\%) at high risk of malnutrition and $16(17 \%)$ at moderate risk. Only $21(22 \%)$ had an NST completed by the nursing staff. Eighteen patients had been prescribed ONS; patterns of prescription compared with nutritional risk and ward documentation of food intake (appetite and ability to eat taken from NST) are shown in Table 1. Compared with the dietitians, the non-dietetic staff prescribed ONS more often in patients at low nutritional risk or where there was no NST completed. The patients consumed more of the prescribed ONS when it was prescribed by a dietitian than by a doctor or a nurse.

Table 1. Patterns of prescription by dietitians, doctors and nurses and nutrition risk in the patients receiving ONS

\begin{tabular}{|c|c|c|c|c|c|c|}
\hline \multirow[b]{2}{*}{ ONS prescribed by } & \multicolumn{3}{|c|}{ No. of patients and level of nutritional risk: } & \multirow[b]{2}{*}{ Total no. (\%) } & \multirow[b]{2}{*}{ NST completed by ward staff } & \multirow[b]{2}{*}{$\%$ of prescribed ONS taken } \\
\hline & High (NST 6-15) & Moderate (NST 4-5) & Low (NST 0-3) & & & \\
\hline Dietitian & 6 & 1 & 1 & $8(44)$ & 8 & 58 \\
\hline Doctor & 1 & 2 & 2 & $5(28)$ & 1 & 44 \\
\hline Nurse & 0 & 4 & 1 & $5(28)$ & 3 & 14 \\
\hline Total & 7 & 7 & 4 & $18(100)$ & 12 & \\
\hline
\end{tabular}

More ONS was prescribed by non-dietetic than dietetic staff and while the rationale for prescription was unclear, non-dietetic prescribing was often in patients who were not at risk of malnutrition and with no evidence that food intake (and thus energy and protein intake) had been considered, so did not comply with NICE guidance. Dietetic prescribing tended to comply with NICE guidance as patients were at risk of malnutrition and had food intake (NST) documented at the ward level and as part of the dietetic assessment. The reason for higher uptake of ONS when prescribed by a dietitian was not assessed but may have been due to dietitians taking the patients' likes and dislikes into account when recommending the type and flavour of ONS. Rates of nutritional screening by ward staff were disappointingly low and similar to previous audits ${ }^{(3)}$.

1. http://guidance.nice.org.uk/CG32

2. Reilly HM, Martineau JK, Moran A et al. (1995) Clin Nutr 14, 269-273.

3. Beresford H, ODonnell M \& Soulsby CT (2008) Proc Nutr Soc 67, E125. 